\title{
Brans-Dicke model constrained from the Big Bang nucleosynthesis and magnitude redshift relations of supernovae (Research Note)
}

\author{
E. P. B. A. Thushari ${ }^{1}$, R. Nakamura ${ }^{1}$, M. Hashimoto ${ }^{1}$, and K. Arai ${ }^{2}$ \\ 1 Department of Physics, Kyushu University, Fukuoka, 812-8581, Japan \\ e-mail: [berni; riou; hashimoto] @phys. kyushu-u.ac.jp \\ 2 Department of Physics, Kumamoto University, Kumamoto, 860-8555, Japan \\ e-mail: arai@sci.kumamoto-u.ac.jp
}

Received 31 March 2010 / Accepted 12 July 2010

\section{ABSTRACT}

\begin{abstract}
We investigated the Brans-Dicke model with a variable cosmological term $(B D \Lambda)$ with the coupling constant $\omega=10^{4}$. We constrained the parameters inherent in this model from a comparison between the Big Bang nucleosynthesis and the observed abundances. Furthermore, we studied the magnitude redshift $(m-z)$ relations for the $B D \Lambda$ with and without another constant cosmological term in a flat universe. Observational data of type Ia supernovae (SNIa) are used in the redshift range of $0.01<z<2$. We found that our model with an energy density of the constant cosmological term of 0.7 can explain the SNIa observations, though the model parameters are insensitive to the $m-z$ relation.
\end{abstract}

Key words. primordial nucleosynthesis - dark energy - early Universe - cosmology: observations - dark matter - cosmology: theory

\section{Introduction}

Astronomical observations indicate that the cosmological constant in the very early universe exceeds the present value by some 120 orders of magnitude, which is estimated in modern theories of elementary particles (Weinberg 1989). This is one of the fine-tuning problems in cosmology called the "cosmological constant problem". To explain the puzzle in cosmology, new modified theories are needed beyond the standard model. The behavior of the cosmological term has motivated various functional forms of the cosmological term. The mechanism of the dynamical reduction of the cosmological term is formulated as a time dependent function (Silviera \& Waga 1997) and in terms of a scalar field (Weinberg 1989; Huterer \& Turner 1999). On the other hand, generalized scalar tensor theories have been investigated (Wagoner 1970; Endo \& Fukui 1977; Fukui et al. 2001).

One of them the Brans-Dicke (BD) theory with a variable cosmological term $(\Lambda)$ as a function of scalar field $(\phi)$ (Endo \& Fukui 1977). This model has been investigated for the early universe and constrained from the Big Bang nucleosynthesis (BBN) (Arai et al. 1987; Etoh et al. 1997; Nakamura et al. 2006) for the coupling constant $\omega \leq 500$. Present observations suggest that the value of $\omega$ exceeds 40000 (Berti et al. 2003; Bertotti et al. 2005). Therefore it is worthwhile to reconstrain the parameters in the Brans-Dicke model with a variable cosmological term $(B D \Lambda)$ for a new value of $\omega$. The $B D \Lambda$ has played a very important role in explaining the characteristics of the early universe (Arai et al. 1987; Etoh et al. 1997; Nakamura et al. 2006). However, we still need an answer to the question "How does this model work at the present epoch?". Therefore we adopt the magnitude redshift $(m-z)$ relations of type Ia supernova (SNIa) observations. This is because the cosmological term significantly affects the cosmic expansion rate of the universe at the low redshifts.
The SNIa observations imply that the universe is accelerating at the present epoch (Perlmutter et al. 1999; Riess et al. 1998, 1999).

In Sect. 2 the formulation of the $B D \Lambda$ is reviewed. Parameters inherent in this model are constrained in Sect. 3 from the Big Bang nucleosynthesis for $\omega=10^{4}$. In Sect. 4 the $m-z$ relation is investigated for the $B D \Lambda$ with including another constant cosmological term in a flat universe. Recent SNIa observational data (Astier et al. 2006; Riess et al. 2007; Kessler et al. 2009) are adopted to constrain the models. Concluding remarks are given in Sect. 5 .

\section{Brans-Dicke model with a variable cosmological term}

The field equations for the $B D \Lambda$ are written as follows (Arai et al. 1987):

$$
\begin{aligned}
R_{\mu \nu}-\frac{1}{2} g_{\mu \nu} R+g_{\mu \nu} \Lambda= & \frac{8 \pi}{\phi} T_{\mu \nu}+\frac{\omega}{\phi^{2}}\left(\phi_{, \mu ; \nu}-\frac{1}{2} g_{\mu \nu} \phi_{, \alpha} \phi^{, \alpha}\right) \\
& +\frac{1}{\phi}\left(\phi_{, \mu ; \nu}-g_{\mu \nu} \square \phi\right), \\
R-2 \Lambda-2 \phi \frac{\partial \Lambda}{\partial \phi}= & \frac{\omega}{\phi^{2}} \phi_{, \nu} \phi^{, v}-\frac{2 \omega}{\phi} \square \phi,
\end{aligned}
$$

where $\phi$ is the scalar field and $T_{\mu \nu}$ is the energy-momentum tensor of the matter field. The Robertson-Walker metric for homogeneous and isotropic universe writes as (Weinberg 1972):

$\mathrm{d} s^{2}=-\mathrm{d} t^{2}+a(t)^{2}\left[\frac{\mathrm{d} r^{2}}{1-k r^{2}}+r^{2} \mathrm{~d} \theta^{2}+r^{2} \sin ^{2} \theta \mathrm{d} \phi^{2}\right]$, 
where $a(t)$ is the scale factor and $k$ is the curvature constant. Here we adopt $c=1$. The expansion is described by the following equation derived from the $(0,0)$ component of Eq. (1):

$$
\left(\frac{\dot{a}}{a}\right)^{2}=\frac{8 \pi \rho}{3 \phi}-\frac{k}{a^{2}}+\frac{\Lambda}{3}+\frac{\omega}{6}\left(\frac{\dot{\phi}}{\phi}\right)^{2}-\frac{\dot{a}}{a} \frac{\dot{\phi}}{\phi},
$$

where $\rho$ is the energy density.

We adopt the simplest case of the coupling between the scalar, and the matter field is

$\square \phi=\frac{8 \pi \mu}{2 \omega+3} T_{v}^{v}$,

where $\mu$ is a constant. Assuming a perfect fluid for $T_{\mu \nu}$, Eq. (5) reduces to

$\frac{\mathrm{d}}{\mathrm{d} t}\left(\dot{\phi} a^{3}\right)=\frac{8 \pi \mu}{2 \omega+3}(\rho-3 p) a^{3}$,

where $p$ is the pressure.

A particular solution of Eq. (2) is obtained from Eqs. (1) and (5):

$\Lambda=\frac{2 \pi(\mu-1)}{\phi} \rho_{\mathrm{m}_{0}} a^{-3}$,

where $\rho_{\mathrm{m}_{0}}$ is the matter density at the present epoch.

The gravitational "constant" $G$ is expressed as

$G=\frac{1}{2}\left(3-\frac{2 \omega+1}{2 \omega+3} \mu\right) \frac{1}{\phi}$.

The density $\rho$ and the pressure $p$ are replaced by

$\rho=\rho_{\mathrm{m}}+\rho_{\gamma}$,

$p=p_{\gamma}=\rho_{\gamma} / 3$,

where the energy density of matter varies as $\rho_{\mathrm{m}}=\rho_{\mathrm{m}_{0}} a^{-3}$. The energy density of radiation is written as $\rho_{\gamma}=\rho_{\gamma_{0}} a^{-4}$ except $\mathrm{e}^{ \pm}$epoch: $\rho_{\gamma}=\rho_{\mathrm{rad}}+\rho_{\nu}+\rho_{\mathrm{e}^{ \pm}}$at $t \leq 1 \mathrm{~s}$, where subscripts rad, $v$ and $\mathrm{e}^{ \pm}$are for photons, neutrinos, and electron-positrons, respectively (Nakamura et al. 2006). The subscript " 0 " indicates the values at the present epoch.

Then, Eq. (6) is integrated to give

$\dot{\phi}=\frac{1}{a^{3}}\left[\frac{8 \pi \mu}{2 \omega+3} \rho_{\mathrm{m}_{0}} t+B\right]$,

where $B$ is an integral constant, and here we use the normalized value of $B: B^{*}=B /\left(10^{-24} \mathrm{~g} \mathrm{~s} \mathrm{~cm}^{-3}\right)$.

The original Brans-Dicke theory is deduced for $\mu=1$ and is reduced to the Friedmann model when $\phi=$ const. and $\omega \gg 1$. Physical parameters have been used to solve Eqs. (4), (7), and (11): $G_{0}=6.6726 \times 10^{-8} \mathrm{~cm}^{3} \mathrm{~g}^{-1} \mathrm{~s}^{-2}, H_{0}=$ $71 \mathrm{~km} \mathrm{~s}^{-1} \mathrm{Mpc}^{-1}$ (Spergel et al. 2003), and $\omega=10^{4}$ (Berti et al. 2003; Bertotti et al. 2005). Figure 1 shows the evolution of the scale factor in the $B D \Lambda$ for the several values of $B^{*}$. We identify considerable deviations in the $B D \Lambda$ from the Friedmann model at $t<100 \mathrm{~s}$, which depends on the specific parameters. Therefore the $B D \Lambda$ should be constrained from the BBN (Arai et al. 1987; Etoh et al. 1997; Nakamura et al. 2006).

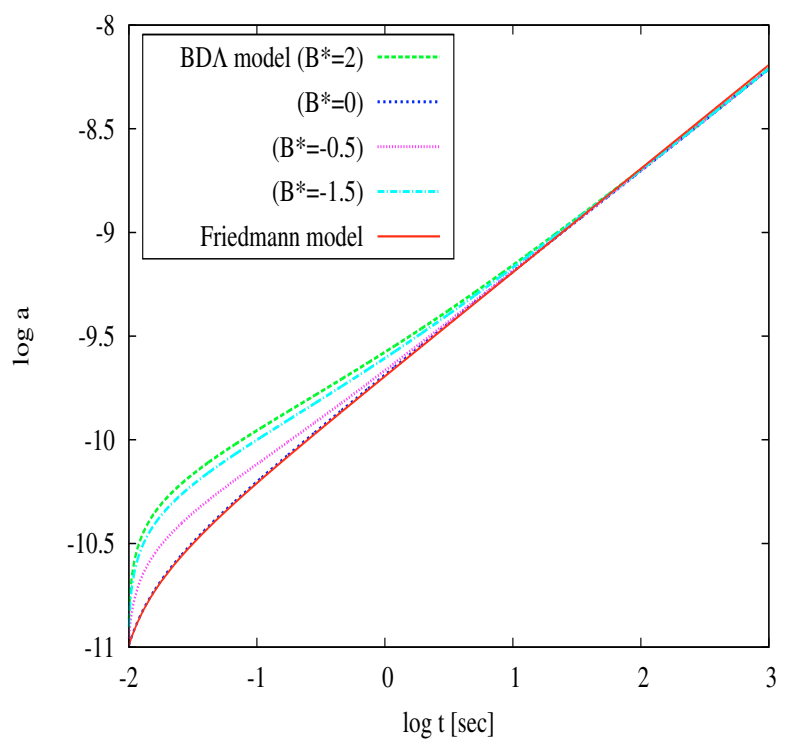

Fig. 1. Time evolutions of the scale factor in the $B D \Lambda$ with $\mu=0.6$ which are compared to the Friedmann model.

\section{Parameters constrained from the Big Bang nucleosynthesis}

The Big Bang nucleosynthesis provides powerful constraints on possible deviation from the standard cosmology (Malaney \& Mathews. 1993). As shown in Fig. 1, the expansion rates of the $B D \Lambda$ differ significantly from those of the standard Friedmann model.

The abundance of light elements in the $B D \Lambda$ has already been investigated (Arai et al. 1987; Etoh et al. 1997; Nakamura et al. 2006). In the previous studies, the parameters inherent in the $B D \Lambda$ have been constrained for $\omega=500$. But we consider the case $\omega=10^{4}$ for convenience, because the Cassini measurements of the Shapiro time delay indicate $\omega \geq 4 \times 10^{4}$ (Berti et al. 2003; Bertotti et al. 2005). The detailed method of nucleosynthesis is described in Nakamura et al. 2006.

Figure 2 shows the calculated abundances of ${ }^{4} \mathrm{He}, \mathrm{D}$, and ${ }^{7} \mathrm{Li}$ for $B^{*}=2$ and $\mu=0.6$. The $\pm 2 \sigma$ uncertainties in nuclear reaction rates are indicated by the dashed lines. The horizontal dotted lines indicate the observational values of ${ }^{4} \mathrm{He}$, $\mathrm{D} / \mathrm{H}$, and ${ }^{7} \mathrm{Li} / \mathrm{H}$ as follows: $Y_{p}=0.2516 \pm 0.0080$ (Fukugita \& Kawasaki 2006; Carr et al. 2010), $Y_{p}=0.326 \pm 0.075$ (Komatsu et al. 2010), $\mathrm{D} / \mathrm{H}=(2.82 \pm 0.21) \times 10^{-5}$ (Pettini et al. 2008), ${ }^{7} \mathrm{Li} / \mathrm{H}=(2.34 \pm 0.32) \times 10^{-10}$ (Melendez \& Ramirez 2004). Here two observational values of ${ }^{4} \mathrm{He}$ are used. The solid vertical lines indicate the WMAP constraint of the baryon-to-photon ratio, $\eta=(6.19 \pm 0.15) \times 10^{-10}$ (Komatsu et al. 2010).

The intersection range of the two observational values of ${ }^{4} \mathrm{He}$ is used to constrain the parameters. We found that the values of $\eta$ derived from ${ }^{4} \mathrm{He}$ and $\mathrm{D} / \mathrm{H}$ are tightly consistent with the value by the WMAP, though the lower limit of ${ }^{7} \mathrm{Li} / \mathrm{H}$ is barely consistent. These agreements lead us to obtain the parameter ranges of $0.0 \leq \mu \leq 0.6$ and $-2 \leq B^{*} \leq 2$.

\section{4. $m-z$ relation in the $B D \wedge$ with and without a constant cosmological term}

The distance modulus $\mu_{\text {th }}$ of the source at the redshift $z$ is

$\mu_{\text {th }}=m-M=5 \log _{10}\left[(1+z) r_{l}\right]+25$, 
E. P. B. A. Thushari et al.: Brans-Dicke model constrained from $m-z$ relation $(R N)$

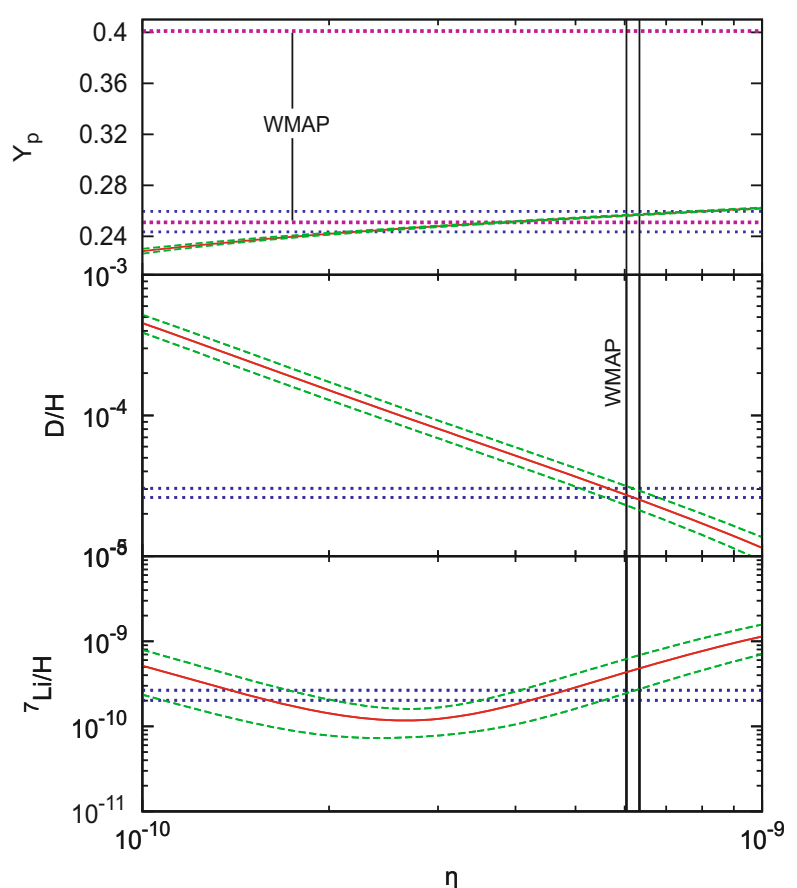

Fig. 2. Light element abundances of ${ }^{4} \mathrm{He}, \mathrm{D}$, and ${ }^{7} \mathrm{Li}$ vs. $\eta$ for $B^{*}=2$, $\mu=0.6$, and $\omega=10^{4}$. Dashed lines indicate the $\pm 2 \sigma$ uncertainties in nuclear reaction rates in each abundance. The horizontal dotted lines indicate the regions of observational abundances. The solid vertical lines indicate the baryon-to-photon ratio $\eta$.

where $m$ and $M$, are the apparent and absolute magnitudes respectively, and $r_{l}$ stands for the radial distance in units of Mpc.

We adopt the SNIa (Astier et al. 2006; Riess et al. 2007; Kessler et al. 2009) for which $\chi^{2}$ is defined by

$\chi^{2}=\sum_{i} \frac{\left(\mu_{\mathrm{th}, i}-\mu_{\mathrm{obs}, i}\right)^{2}}{\sigma_{i}^{2}}$,

where $\mu_{\mathrm{th}, i}$ is given by Eq. (12), $\mu_{\mathrm{obs}, i}$ and $\sigma_{i}$ are the observed values of distance modulus and their uncertinities.

For the homogeneous and isotropic universe, the relation between the radial distance and the redshift is derived from the Robertson-Walker metric as (Weinberg 2008)

$\int_{0}^{z} \frac{\mathrm{d} z}{H}= \begin{cases}k^{-1 / 2} \sin ^{-1}\left(\sqrt{k} r_{l}\right) & k=+1, \\ r_{l} & k=0, \\ |k|^{-1 / 2} \sinh ^{-1}\left(\sqrt{|k|} r_{l}\right) & k=-1,\end{cases}$

where $H=\dot{a} / a$ is the expansion rate written from Eq. (4) as

$H=\left[\frac{1}{4}\left(\frac{\dot{\phi}}{\phi}\right)^{2}-(1+z)^{2} k+\frac{\Lambda}{3}+\frac{\omega}{6}\left(\frac{\dot{\phi}}{\phi}\right)^{2}+\frac{8 \pi}{3} \frac{\rho}{\phi}\right]^{\frac{1}{2}}-\frac{1}{2} \frac{\dot{\phi}}{\phi}$.

We conclude from the WMAP results that we live in a closely geometrically flat universe (Dunckley et al. 2009). The present matter density $\rho_{\mathrm{m}_{0}}$ is obtained from Eq. (4) as

$H_{0}^{2}=\frac{1}{3}\left(\frac{8 \pi \rho_{\mathrm{m}_{0}}}{\phi_{0}}+\Lambda_{0}\right)+\frac{\omega}{6}\left(\frac{\dot{\phi}}{\phi}\right)_{0}^{2}-\left(\frac{\dot{\phi}}{\phi} H\right)_{0}$,
$\rho_{\mathrm{m}_{0}}=4 \rho_{\mathrm{c}}^{B D \Lambda} /(\mu+3), \rho_{\mathrm{c}}^{B D \Lambda}=3 \phi_{0} H_{0}^{2} / 8 \pi$,

where the $\rho_{\mathrm{c}}^{B D \Lambda}$ is the critical density of $B D \Lambda$.

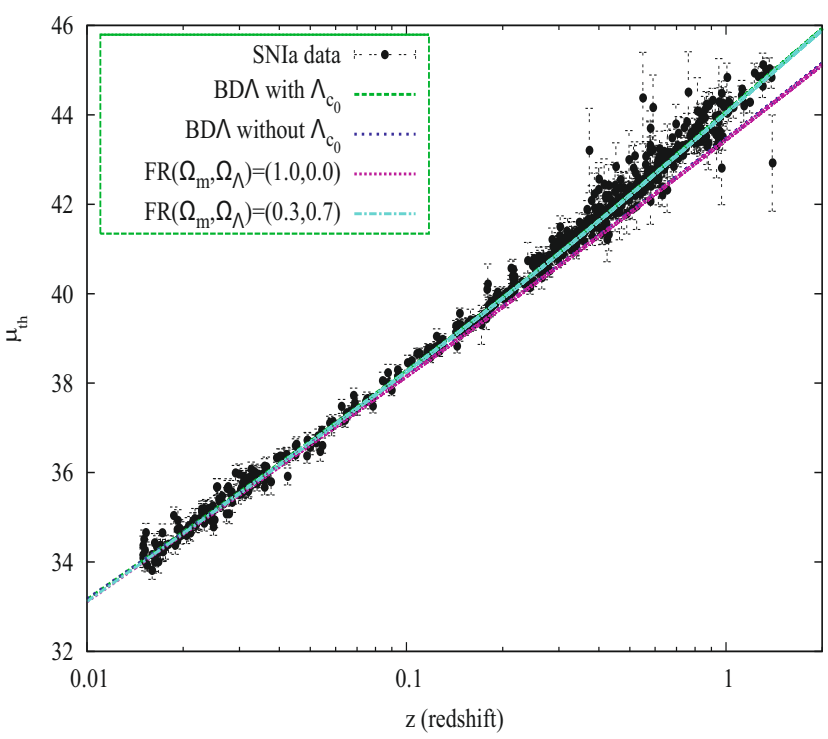

Fig. 3. Distance modulus vs. redshift for the flat universe in the Friedmann model and the $B D \Lambda$ with and without constant cosmological term constrained by the SNIa observations (Riess et al. 2007; Astier et al. 2006; Kessler et al. 2009).

Using the analogy with the Lemaittre model, Eq. (15) is transformed as

$\Omega_{\mathrm{m}_{0}}+\Omega_{\Lambda_{0}}+\Omega_{\phi_{0}}=1$.

Here, the energy density parameters are defined as

$\Omega_{\mathrm{m}_{0}}=\frac{\rho_{\mathrm{m}_{0}}}{\rho_{\mathrm{c}}^{B D \Lambda}}, \Omega_{\Lambda_{0}}=\frac{(\mu-1) \rho_{\mathrm{m}_{0}}}{4 \rho_{\mathrm{c}}^{B D \Lambda}}$,

$\Omega_{\phi_{0}}=\frac{\omega}{6 H_{0}^{2}}\left(\frac{\dot{\phi}}{\phi}\right)_{0}^{2}-\left(\frac{\dot{\phi}}{\phi} \frac{1}{H_{0}}\right)_{0}$.

The value $\Omega_{\phi_{0}}$ is found to be very low as $7.01 \times 10^{-5}$ for $\mu=$ 0.6. If we consider the absolute value of $\Omega_{\phi_{0}}$ in the parameter range $0.0 \leq \mu \leq 0.6$, its contribution to Eq. (17) is always less than $10^{-5}$. Therefore, as long as we consider the present epoch, contribution from $\Omega_{\phi_{0}}$ can be neglected.

Figure 3 shows the $m-z$ relation in the $B D \Lambda$ for the SNIa observations. Matter is dominant in this model. The energy density of the cosmological term is always less than $20 \%$ in the best-fit parameter region predicted in Sect. 3 . The energy density of the cosmological term always takes negative values in the obtained parameter region. The parameter $B^{*}$ is not effective to change the values of $\Omega_{\mathrm{m}_{0}}$ and $\Omega_{\Lambda_{0}}$. Because this model is matter dominant, it cannot be constrained by the SNIa observations.

The Friedmann model with the energy density parameters of $\left(\Omega_{\mathrm{m}}, \Omega_{\Lambda}\right)=(1.0,0.0)$ is merged into the $B D \Lambda$ model with the reduced $\chi_{\mathrm{r}}^{2} \equiv \chi^{2} / N \simeq 4.117$ (where $\chi^{2}=2293$ and $N$ is defined as degrees of freedom). This is inconsistent with the present accelerating universe, which should contain a sufficient amount of dark energy to accelerate the universe. To explain the present accelerating universe, we need some modification of the cosmological term.

As the next approach, the $B D \Lambda$ is modified by adding another constant cosmological term $\Lambda_{\mathrm{c}_{0}}$. The expansion rate in this model is written by

$H=\left[\frac{1}{4}\left(\frac{\dot{\phi}}{\phi}\right)^{2}+\frac{\Lambda}{3}+\frac{\Lambda_{\mathrm{c}_{0}}}{3}+\frac{\omega}{6}\left(\frac{\dot{\phi}}{\phi}\right)^{2}+\frac{8 \pi}{3} \frac{\rho}{\phi}\right]^{\frac{1}{2}}-\frac{1}{2} \frac{\dot{\phi}}{\phi}$. 
The present matter density is

$\rho_{\mathrm{m}_{0}}=\frac{4\left(1-\Lambda_{\mathrm{c}_{0}}\right) \rho_{\mathrm{c}}^{B D \Lambda}}{(\mu+3)}$.

Here the energy density parameter of the constant cosmological term is fixed to be 0.7 .

We find that this model is consistent with the SNIa observations as seen in Fig. 3. The total cosmological term becomes large in this model and consistent with the present accelerating universe with reduced $\chi_{\mathrm{r}}^{2} \simeq 0.98$ (where $\left.\chi^{2}=546.92\right)$. For $\mu=0.5$, the $B D \Lambda$ with $\Lambda_{\mathrm{c}_{0}}$ predicts $\Omega_{\Lambda}=-4.285 \times 10^{-2}$ and $\Omega_{\mathrm{m}}=0.343 . \Omega_{\Lambda}$ always gets negative values in the parameter region of $\mu$ predicted in Sect. 3. If we consider the total value of energy densities, the contribution from $\Omega_{\Lambda_{0}}+\Omega_{\Lambda_{c_{0}}}$ to the total energy density is always between $60-67 \%$. Therefore the cosmological term is dominant in the present epoch and can be constrained from the present SNIa observations. We conclude that the $B D \Lambda$ with $\Lambda_{\mathrm{c}_{0}}$ has nearly the same energy density parameters as the Friedmann model with $\left(\Omega_{\mathrm{m}}, \Omega_{\Lambda}\right)=(0.3,0.7)$. Although the cosmological term is not important at the early epoch, it plays a very important role at the present era. All the parameters inherent in the $B D \Lambda$ become insufficient as far as the $m-z$ relation is concerned.

\section{Concluding remarks}

Previous BBN calculations restricted the parameter range as $-0.5 \leq \mu \leq 0.8$ and $-10 \leq B^{*} \leq 10$ for $\omega=500$ (Nakamura et al. 2006). On the other hand, our high value of $\omega=10^{4}$ leads to reduces the parameter range of $B^{*}\left(-2 \leq B^{*} \leq-2\right)$ and $\mu$ $(0.0 \leq \mu \leq 0.6)$. The models parameters are inefficient in the $m-z$ relations of the SNIa.

In Sect. 4 , the value of $\Omega_{\phi_{0}}$ is found to be much smaller compared with the other terms in Eq. (17). Even though $\omega$ is increased until $10^{4}$, the contribution from $\Omega_{\phi_{0}}$ to Eq. (17) is always less than $1 \%$ in that particular parameter range. There is no considerably negative effect from the assumption we made in Sect. 4 to neglect the value of $\Omega_{\phi_{0}}$. In the parameter range $0.0 \leq \mu \leq 0.6, \Lambda$ has taken negative values according to Eq. (17). This may not conflict with theories, because the pressure of dark energy must be negative to reproduce the present accelerated expansion (Carroll 1992).

Note that from Eq. (7), $\Lambda \sim \rho_{\mathrm{m}} / \phi$ and at the present epoch, $\Lambda_{0}$ is directly connected with $\rho_{\mathrm{m}_{0}}$. Dark energy is written in terms of dark matter. However, dark energy and dark matter should be distinguishable to give rise to an accelerated expansion, because the evolution of the scale factor critically depends on the composition of each energy density of the universe. Therefore, the $B D \Lambda$ without a constant cosmological term is indistinguishable from the matter-dominant Friedmann model with the parameters of $\left(\Omega_{\mathrm{m}}, \Omega_{\Lambda}\right)=(1.0,0.0)$. Note that the variable $\Lambda$ term in the $B D \Lambda$ plays a minor role to accelerate the universe at the present epoch. Because of this, we modified $\Lambda$ by adding a constant cosmological term. It has no relation to the expansion rate of the universe at the early epoch. However, the energy stored in the constant cosmological term played a major role in accelerating the universe at the present epoch as seen in Fig. 3. Since this model contains enough dark energy to accelerate the universe, it is constrained by the SNIa observations. In the present research, we investigated the $B D \Lambda$ at the early epoch to determine the intrinsic parameters and introduce new parameters at the present epoch for the $m-z$ relation. Because we demonstrated a possibility of a non-standard model which is compatible with the observations, it is worthwhile to examine more generally the functional form of the cosmological term (e.g. Fukui et al. 2001).

\section{References}

Arai, K., Hashimoto, M., \& Fukui, T. 1987, A\&A, 179, 17

Astier, P., Guy, J., Regnault, N., et al. 2006, A\&A, 447, 31

Berti, E., Buonanno, A., \& Will, C. M. 2005, Phys. Rev. D, 71, 084025

Bertotti, B., Iess, L., \& Tortora, P. 2003, Nature, 425, 374

Carr, B. J., Kohri, K., Sendouda, Y., \& Yokoyama, J., et al. 2010, Phys. Rev. D, 81,104109

Carroll, S. M., \& Press, W. H. 1992, ARA\&A, 30, 499

Dunckley, J., Komatsu, E., Nolta, M. R., et al. 2009, ApJS, 180, 306 Endo, M., \& Fukui, T. 1977, Gen. Rel. Grav., 8, 833

Etoh, T., Hashimoto, M., Arai, K., \& Fujimoto, S. 1997, A\&A, 325, 893

Fukugita, M., \& Kawasaki, M. 2006, ApJ, 646, 691

Fukui, T., Arai, K., \& Hashimoto, M. 2001, Class. Quant. Grav., 18, 2087

Huterer, D., \& Turner, M. S. 1999, Phys. Rev. D, 60, 081301

Kessler, R., Becker, A. C., Cinabro, D., et al. 2009, ApJS, 185, 32

Komatsu, E., Smith, K. M., Dunkley, J., et al. 2010, ApJS, submitted [arXiv: $1001.4538 \mathrm{v} 2]$

Malaney, R. A., \& Mathews, G. J. 1993, Phys. Rep., 229, 145

Melendez, J., \& Ramirez, I. 2004, ApJ, 615, L33

Nakamura, R., Hashimoto, M., Gamow, S., \& Arai, K. 2006, A\&A, 448, 23

Perlmutter, S., Aldering, G., Goldhaber, G., et al. 1999, ApJ, 517, 565

Pettini, M., Zych, B. J., Murphy, M. T., Lewis, A., \& Steidel, C. C. 2008, MNRAS, 391, 1499

Riess, A. G., Filippenko, A. V., Challis, P., et al. 1998, AJ, 116, 1009

Riess, A. G., Kirshner, R. P., Schmidt, B. P., et al. 1999, AJ, 117, 707

Riess, A. G., Strolger, L.-G., Casertano, S., et al. 2007, ApJ, 659, 98

Silviera, V., \& Waga, I. 1997, Phys. Rev. D, 56, 4625

Spergel, D. N., Verde, L., Peiris, H. V., et al. 2003, ApJS, 148, 175

Weinberg, S. 1972, Gravitation and Cosmology (New York: John Wiley)

Weinberg, S. 1989, Rev. Mod. Phys., 61, 1

Weinberg, S. 2008, Cosmology (New York: Oxford Unversity Press)

Wagoner, R. V. 1970, Phys. Rev. D, 1, 3209 